\title{
ENERgy EFFiCiENT Military Mobile BASE Station Placement
}

\author{
Thabet Mismar, Khaled Elleithy, and Saikat Ray* \\ Department of Computer Sceince and Engineering \\ University of Bridgeport \\ \{tmismar, elleithy\}@bridgeport.edu \\ *raysaikat@gmail.com
}

\begin{abstract}
This research deals with an energy efficient network for military mobile base station placement. The proposed method is based on minimizing the energy loss of military communication networks where the base station is moving along a preset path and the users are constantly moving in an independent speed and direction. It takes into account the free space loss and the knife edge effect for the energy loss to establish a path weight for the shortest path model. Then, it evaluates the neighboring points to the base station for the energy loss of the network in order to find the position at which the minimum energy loss occurs. The results show a clear energy saving advantage when compared to Lloyd-Max's method.
\end{abstract}

\section{KEYWORDS}

Energy Efficiency, Mobile Base Station Placements, Wireless Network, Mobile Network

\section{INTRODUCTION}

Mobile and wireless communication have advanced greatly in the past two decades. Base station placement is the objective of many researchers in the field of wireless sensor networks and the field of mobile networks. Previous research dealt with optimizing the network placement and coverage while considering either the base stations or the users as the moving elements.

The demand for energy efficient communication networks is rising and needed by the concerned users; military demand is not an exception. Energy efficiency is a research objective for environmental and performance reasons, using less energy for a greener planet and enhancing the energy usage of a wireless sensor or a mobile device without the need for recharging.

Mobile base station placement research has mainly considered coverage. It does not emphasize on energy efficiency partly because the focus is on large scale networks and partly because civilian rechargeable mobile devices are not restricted to limited energy. Although there has been a lot of research in the field of base station placement [1-4], there are only a few that focused on mobile base station [5-7].

The situation of both the base station and the users being in constant movement arises in military communication, where energy efficiency is an important issue for most of in-the-field military communication networks.

Extensive studies have been made to develop the best base station placement algorithms. Base station placement algorithms for mobile networks were proposed by [2-4, 8]. Although these algorithms minimize dead zones and increase SNR, they do not take the users' limited energy resources into account. However, energy efficiency is a critical factor for in-the-field military communication networks. 
Fixed base station placement algorithms for wireless sensor networks were proposed by $[1,9]$. Although these algorithms take users into account, they assume a fixed base station for the network which overloads and drains the closest user (sensor) to the base station.

Mobile base station placement was discussed in [5-6, 10]. In [5] the mobile base station placement algorithm was based on the boundary of the transmission range for sensors. This technique uses the limited transmission range for the sensors which does not serve in-the-field military networks.

Algorithms that are based on the transmission range of the sensors and the boundary of the sensors' field were proposed by $[6,10]$. These algorithms perform satisfactorily for sensor networks when the boundary is clearly defined.

Lloyd-Max's method [11] was modified by [12] and used by [7, 13] to develop base station placement algorithms. In [7] the mobile base station placement was based on maximizing network coverage.

The objective of this research is minimizing the energy loss of military communication networks where the base station is moving along a preset path and the users are constantly moving in an independent speed and direction.

The proposed method takes into account the free space loss and the knife edge effect for the energy loss to establish a path weight for the shortest path model and uses Dijkstra's algorithm [14] to determine the shortest path. Then, it evaluates the neighboring points to the base station for the energy loss of the network in order to find the position at which the minimum energy loss occurs.

Lloyd-Max's method for base station placement where the base station is basically centered in the middle of a group of users [7-13] is considered for the purpose of comparing the results. The random waypoint model [15] is used to provide a user movement model where each user is moving around in an independent speed and direction.

\section{Energy EfFicient Mobile Base Station Placement}

In modern warfare, one of the most important elements of winning the war is communication between soldiers and the base command and among the soldiers themselves.

Due to the fact that building bases is not practical, soldiers are constantly moving and construction resources are expensive, a need for mobile base stations arises. Placing the base stations on the battle field in an appropriate place is crucial because the soldier's communication device has a limited energy source and it is essential for it to operate as long as possible.

The objective is to find the new locations of the base stations $(\mathrm{LBi}+1)$ that minimize the total energy lost (TE) in communication, given a set of base stations (BS) and a set of users (MS) at certain locations (LBi) and (LM), consecutively.

One way is just assigning the base station to a random point, which will mean a high energy loss for a few user stations. Another approach is fixing the mobile station to the middle of a group; this approach was first conceived as the Lloyd-Max method and used in [7] which is later compared to the proposed method of mobile base station placement.

The flowchart in Figure 1 shows the evaluation process of the total energy loss of the whole network. The packet routing model uses the user and base station locations in the channel energy loss model to determine the new locations for the base stations. The new base stations locations are then used with the new locations of the users, from the user movement model, in a new round to determine the total energy loss for the whole network in the final round. 


\section{Simulation}

At the beginning the maximum speed for the base station was set to $60 \mathrm{~m} / \mathrm{s}$ [16]. The possible jumps were set to 16 points in order to find the optimum point for the next mobile base station location.

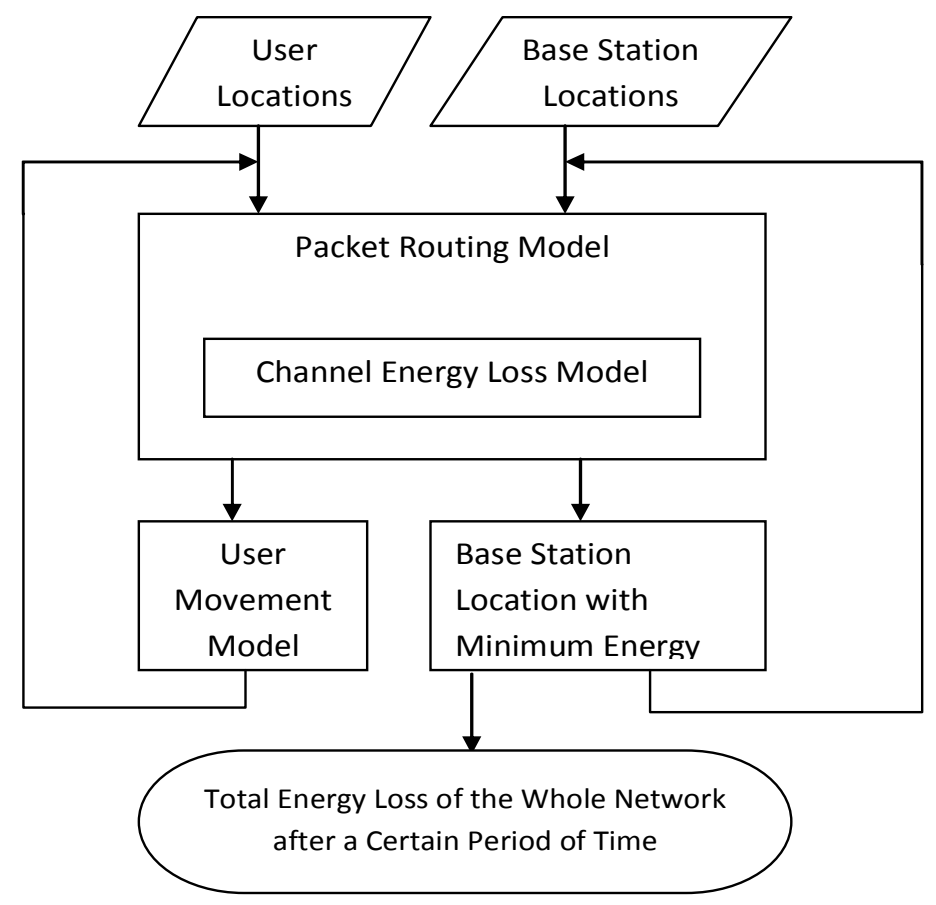

Figure 1. The Problem Flowchart

The models, which are given in this section, clarify the steps taken to move the users, to route the messages to the base station, and to calculate the total energy loss for the users after each possible jump. The downlink communication is ignored for simplicity.

The flowchart in Figure 2 shows the technique used to move the users and the base stations based on the user movement model and the total energy lost in that round. The user locations, the base station locations and the area elevation are the input of Shortest Path First (SPF) which outputs the total energy loss for a certain base station location. The main output of the whole process is the total energy loss for the whole network, which is collected from the total energy losses of each round.

A model for node movement was needed to simulate a large network movement scheme. The model must allow each node to move freely at its own speed to an independent destination. Consequently, the random waypoint model [15] was utilized for user movement.

In the random waypoint model, each node sets a destination and moves to it with a predetermined speed chosen from a minimum-maximum speed range. The users are assumed to be limited in movement by an area of $940 \mathrm{~m}$ x $320 \mathrm{~m}$. Likewise, the speed is chosen randomly to be between $0.4 \mathrm{~m} / \mathrm{s}$ and $1.4 \mathrm{~m} / \mathrm{s}$ [17].

After reaching its destination the node pauses for a period of time, then it sets a new destination with a new speed. A maximum pause period of 5 minutes is assumed.

Packet routing is very important when talking about energy efficient communication systems. Dijkstra's algorithm is an excellent tool to find the shortest path tree for packet routing. 
Assuming that the energy loss to be the edge weight in the algorithm helps achieve energy efficient routing.

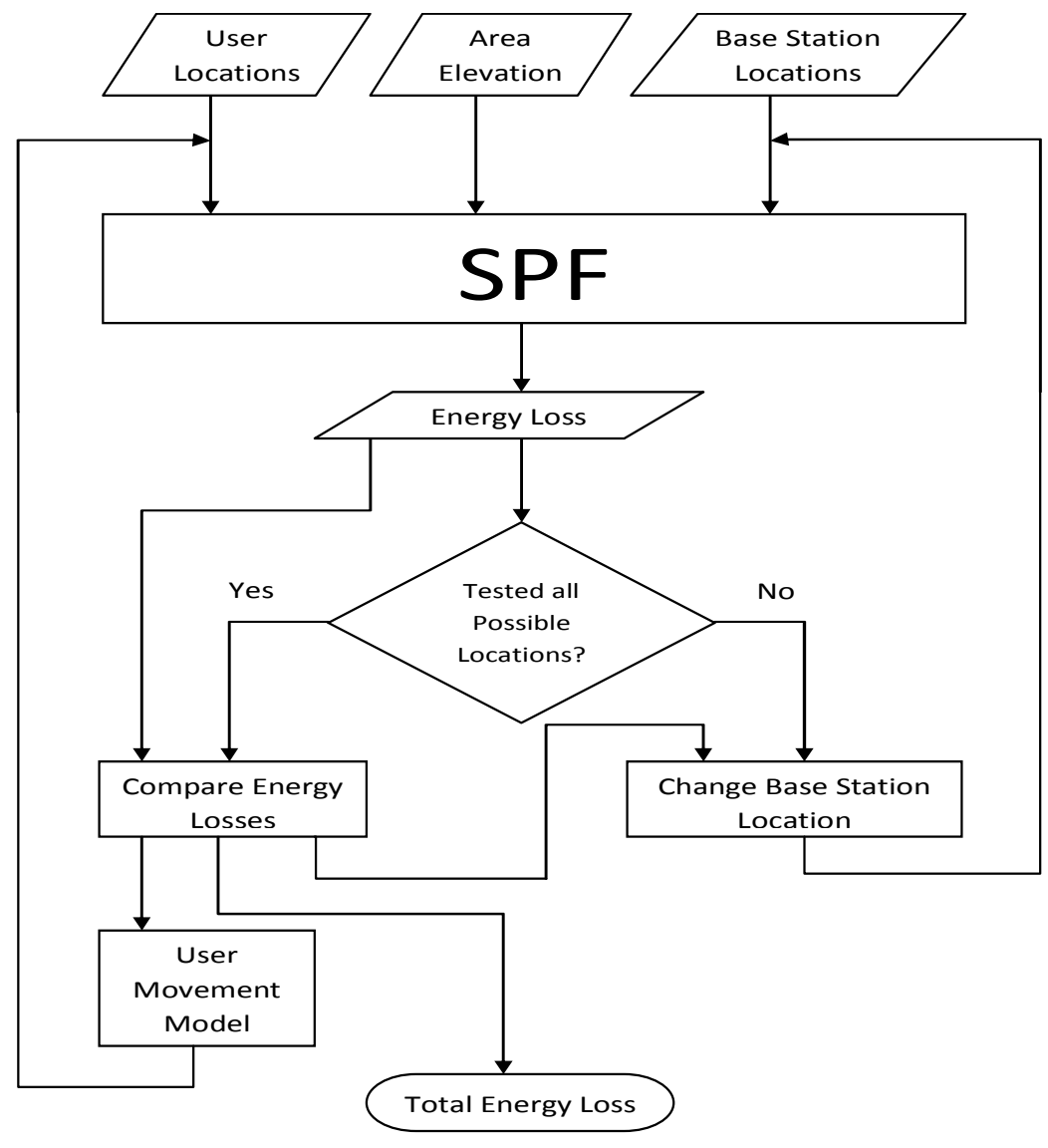

Figure 2. The Flowchart for the Main Program for the Proposed Method (Main.m)

The untested paths are set to infinity, and their weights are set as a percentage of energy lost compared to the total energy of the user. The base station energy loss is not taken into account since it is assumed that the base station has infinite energy reserve and the downlink paths are set to zero, unused paths. After applying Dijkstra's algorithm the path matrix consists of ones and zeros. Zero means the path was not used and one means the path was used to route the packet. A dummy base station is used to eliminate the need for base stations to connect.

The flowchart in Figure 3 shows the technique used to obtain the total energy loss based on Dijkstra's algorithm. The inputs that feed into Free Space Path Loss (FSPL) are the user location, the base station locations and the area elevation. FSPL outputs the path energy losses which are used in Dijkstra's algorithm as the path weights. The output of Dijkstra's algorithm is a spanning tree with the lowest energy path for packet routing, which in turn enables the calculation of the total energy loss for that run.

As mentioned previously, the expected energy losses for the users are used as path weights. The heights of the user and base station antennas are assumed to be $1.5 \mathrm{~m}$ and $100 \mathrm{~m}$, respectively. The free space propagation model is used instead of the two-ray ground reflection model since the distance restriction does not apply in the assumed case [18], i.e. 


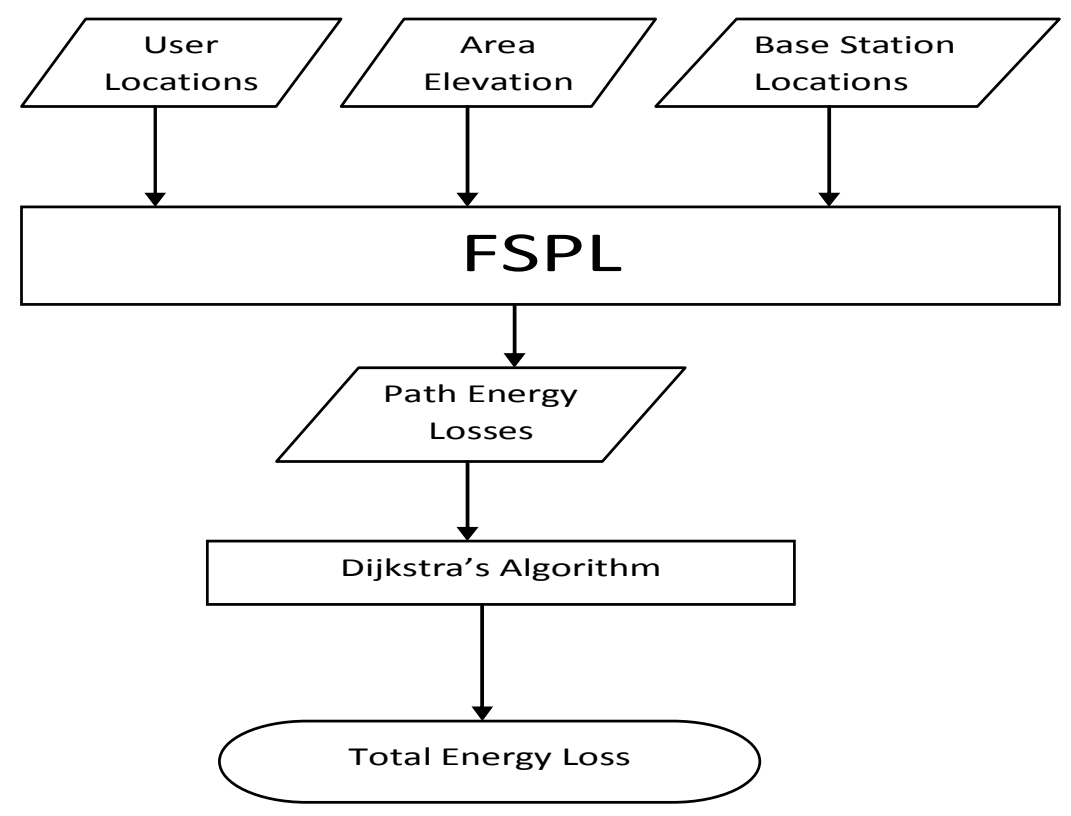

Figure 3. The Flowchart for the Packet Routing Program (SPF.m)

$$
d<\sqrt{h_{t} h_{r}}
$$

where $d$ is the distance between the transmitter and receiver, $h_{t}$ is the antenna height of the transmitter, and $\mathrm{h}_{\mathrm{r}}$ is the antenna height of the receiver.

The following values are assumed for no specific reason:

1) Temperature $=35^{\circ} \mathrm{C}$

2) Channel capacity $=1 \mathrm{Mbps}$

3) $\quad$ Bandwidth $=5 \mathrm{MHz}$

4) $\mathrm{SNR}=10 \mathrm{db}$

The carrier frequency was assumed to be $2.4 \mathrm{GHz}$ due to the fact that the communication channel is based on 802.11. The packet size was set to 4000 bits [19] and the antenna gain for both the transmitter and receiver was set to unity [18].

The knife edge loss is assumed to happen once. Given the current locations of the users and the locations of the base stations, the knife edge loss is calculated by taking pairs of transmitters and receivers and finding the obstructions in the way of the signal between them. Since it is assumed that the knife edge loss happens once, the search is terminated once the first obstruction is found and the loss is calculated using the following equations:

$$
\begin{aligned}
& G_{d}(d B)=20 \log |F(v)| \\
& v=h \sqrt{\frac{2\left(d_{1}+d_{2}\right)}{\lambda d_{1} d_{2}}}
\end{aligned}
$$

where $h$ is the effective height of obstruction, $d_{1}$ and $d_{2}$ are the distances from the obstruction to the transmitter and receiver, and $\lambda$ is the wavelength.

In each round, each base station has $\mathbf{J}$ possible future positions or jumps. For each jump, the expected energy losses for each user $\left(\mathrm{E}_{\mathrm{i}}\right)$ are calculated by 


$$
\begin{aligned}
& \lambda=C / f_{c} \\
& N=K^{*} T * B \\
& P_{R x}=N * S N R \\
& P_{T x}=P_{R x} *(4 \pi * D / \lambda) / L_{k n i f e} \\
& E_{i}=P_{T x} * b / C_{p}
\end{aligned}
$$

where $\lambda$ is the wave length, $C$ is the speed of light, $f_{c}$ is the carrier frequency, $N$ is the white noise, $\mathrm{K}$ is Boltzmann's constant, $\mathrm{T}$ is the temperature in Kelvin, $\mathrm{B}$ is the Bandwidth, $\mathrm{P}_{\mathrm{Rx}}$ is the received transmitted power, $\mathrm{SNR}$ is the signal to noise ratio, $\mathrm{P}_{\mathrm{Tx}}$ is the transmitted power, $\mathrm{D}$ is the distance the signal travels from the transmitter to the receiver, $\mathrm{L}_{\text {knife }}$ is the knife edge loss, $\mathrm{b}$ is the number of bits sent, and $\mathrm{C}_{\mathrm{p}}$ is the channel capacity.

The energy losses $E_{i}$ is then used in Dijkstra's algorithm as the path weights. After applying Dijkstra's algorithm, an estimation of the total network energy loss for a specific possible jump is obtained. This is done for each base station and for all possible jumps in order to take the combination of base station jumps with minimum total energy loss.

The flowchart in Figure 4 shows the technique used to obtain the transmission power. The user locations and the base station locations are used to obtain the distance matrix, which is basically a matrix whose values are the distances between pairs of users or users and base stations. The distance matrix is then used to calculate the transmission power needed before considering the knife edge loss. The knife edge loss is calculated by using the distance matrix and the area elevation. The transmission power and the knife edge loss are finally used to calculate the final transmission power.

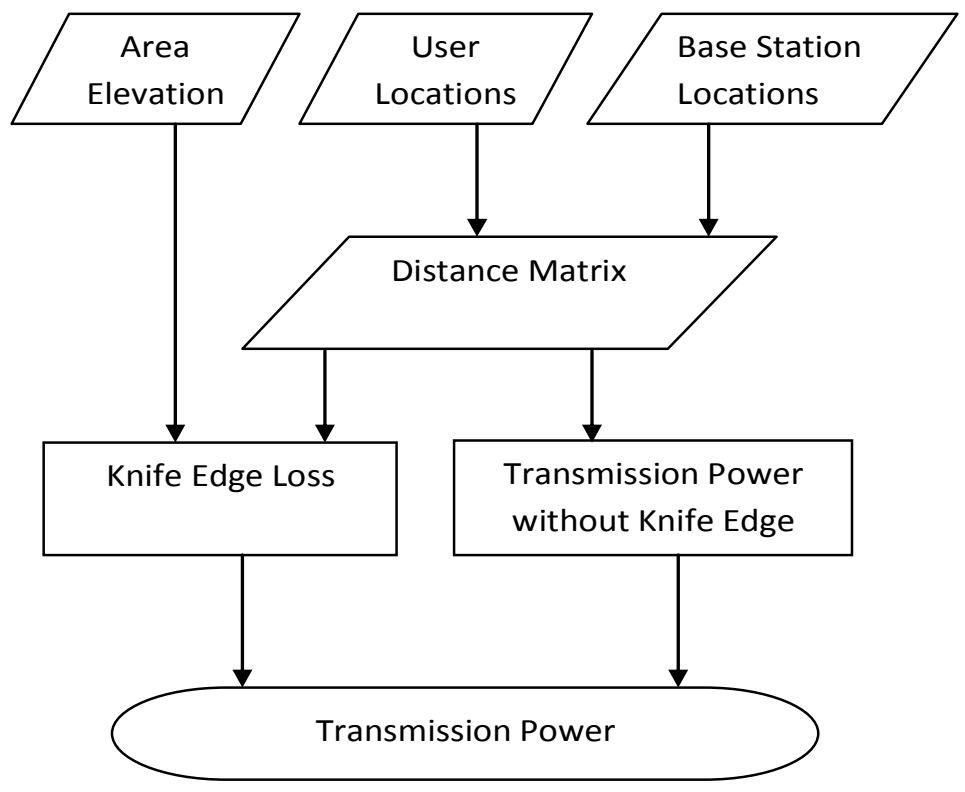

Figure 4. The Flowchart for the Channel Energy Loss Program

(FSPL.m) 


\section{RESUlts}

The simulation mainly focused on varying three parameters; the number of base stations, the number of users, and the average number of packets sent per second.

For each parameter changed, the total energy used by users was calculated for the proposed method and Lloyd-Max's method.

The number of base stations was varied between 1 base station and 5 base stations. Figure 5 shows that the total energy lost by implementing the proposed method and Lloyd-Max's method decreases as the number of base stations increases. This result is expected because as the number of base stations increases the distance between the base station and the users decreases, thus the power needed for transmission is decreased.

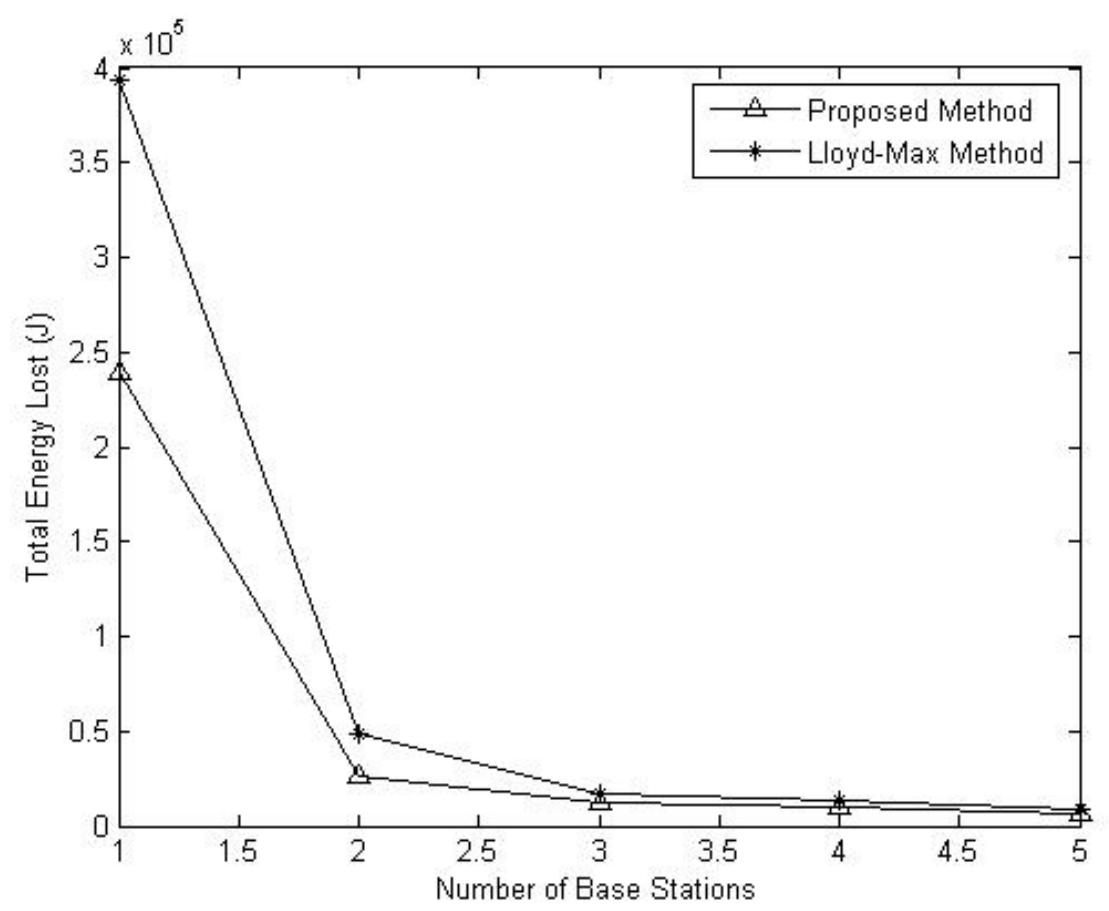

Figure 5. Total Energy Loss for Both Methods. Number of Users $=40$, Average

$$
\text { Packets Sent }=1 \text {. }
$$

The number of users was varied between 20 users and 100 users. Figure 6 shows that the total energy lost by implementing the proposed method and Lloyd-Max's method increases as the number of users increases. This is due to the increase in the number of packets sent through the network.

The number of average packets sent per second was varied between 1 packet and 5 packets per second. Figure 7 shows that the total energy lost by implementing the proposed method and Lloyd-Max's method increases as the number of average packets sent per second increases. This result is expected because the energy lost in transmission is proportional to the number of packets sent. 


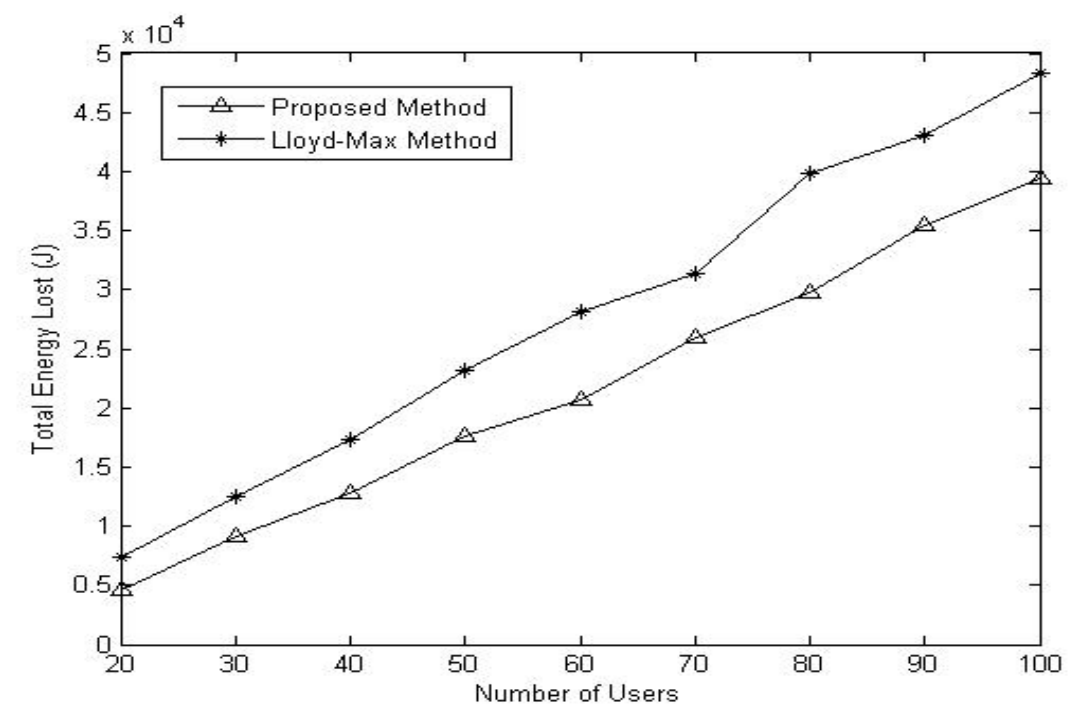

Figure 6. Total Energy Loss for Both Methods. Number of Base stations $=3$, Average Number of Packets $=1$.

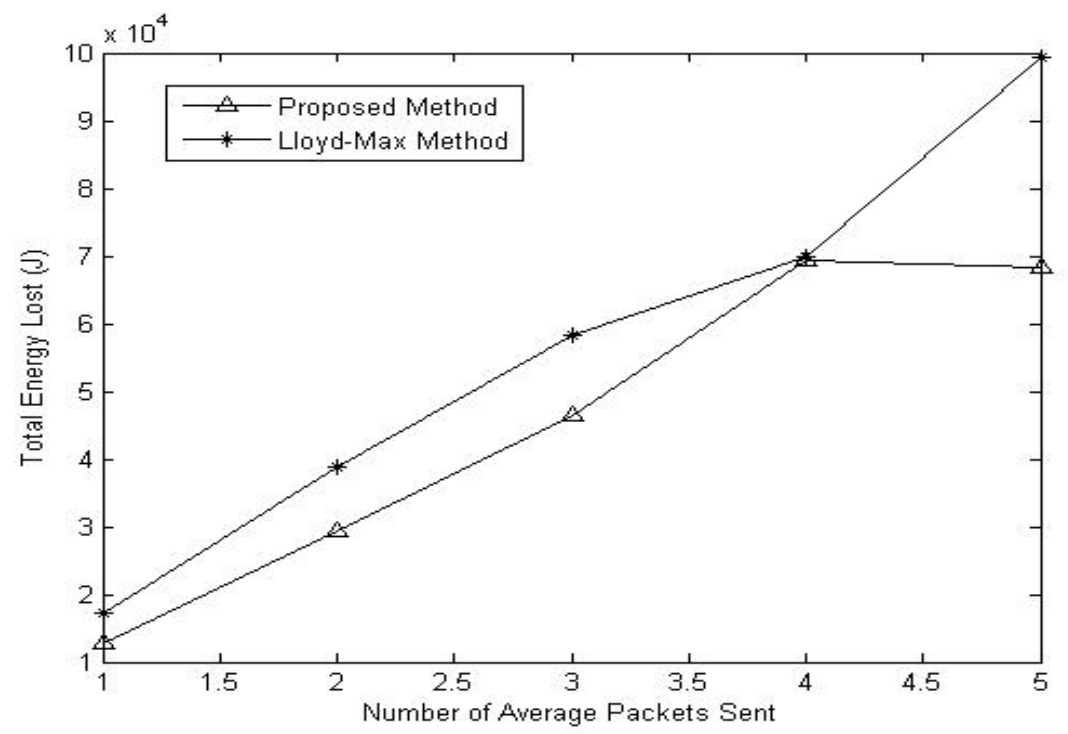

Figure 7. Total Energy Loss for Both Methods. Number of Base stations = 3, Number of Users $=40$.

\section{CONCLUSION}

This research achieved an algorithm for an energy efficient network for military mobile base station placement. The proposed method is based on minimizing the energy loss of military communication networks where the base station is directed to move along an estimated path and the users are constantly moving in an independent speed and direction. The achieved results for the number of base stations, users, and average packets sent indicate that the proposed method is 
suitable for in-the-field military communication networks. Furthermore, the results show a clear average energy saving advantage of $25 \%$ when compared to Lloyd-Max's method.

\section{REFERENCES}

[1] Y. Shi, and Y.T. Hou, "Approximation algorithm for base station placement in wireless sensor networks," in Proc. IEEE Communications Society Conference on Sensor, Mesh and Ad Hoc Communications and Networks (SECON), pp. 512-519, June 18-21, 2007, San Diego, CA, USA.

[2] X. Huang, U. Behr, and W. Wiesbeck. Automatic base station placement and dimensioning for mobile network planning. In IEEE Vehicular Technology Conference, volume 4, pages 1644-$1649,2000$.

[3] B.-S. Park, J.-G. Yook, H.-K. Park, The determination of base station placement and transmit power in an inhomogeneous traffic distribution for radio network planning, in: Proceedings of the IEEE Vehicular Technology Conference, 2002, pp. 2051-2055.

[4] Amaldi, E., Capone, A. and Malucelli. F. (2002) 'Planning UMTS base station location: optimization models with power control and algorithms', IEEE Transactions on Wireless Communications.

[5] Waleed Alsalih, Selim Akl, and Hossam Hassanein, "Placement of multiple mobile base stations in wireless sensor networks,"Proceedings of the IEEE Symposium on Signal Processing and Information Technology (ISSPIT), 2007

[6] Azad, A.P.; Chockalingam, A., Mobile base stations placement and energy aware routing in wireless sensor networks, Wireless Communications and Networking Conference, 2006. WCNC 2006. IEEE Volume 1, 3-6 April 2006. Page(s):264 - 269

[7] Ha Yoon Song. A method of mobile base station placement for High Altitude Platform based network with geographical clustering of mobile ground nodes. Proceedings of the International Multiconference on Computer Science and Information Technology, Oct. 2008. Page: 869-876

[8] Huang, X.; Behr, U.; Wiesbeck, W., A new approach to automatic base station placement in mobile network, Proceedings of the 2000 International Zurich Seminar on Broadband Communications, 2000.

[9] Alon Efrat, Y. Shi and Y. T. Hou, "Algorithm Design for Base Station Placement Problems in Sensor Networks", in 3rd Int. Conf. on Quality of Service in Heterogeneous Wired/Wireless Networks, 2006

[10] S. Gandham, M. Dawande, R. Prakash, and S. Venkatesan, "Energy efficient schemes for wireless sensor networks with multiple mobile base stations," in Proc. The IEEE Global Telecommunications Conference (GLOBECOM), December 2003.

[11] Stuart P. Lloyd. Least Squares Quantization in PCM. IEEE Transactions on Information Theory, vol. 28, no. 2, pp. 129-147, 1982.

[12] Vance Faber, "Clustering and the Continuous K-means Algorithm," Los Alamos Science, No. 22, 1994.

[13] Nirupama Bulusu, John Heidemann and Deborah Estrin, Adaptive Beacon Placement, Proceedings of the Twenty First International Conference on Distributed Computing Systems (ICDCS-21), Phoenix, Arizona, April 2001

[14] Cormen, Thomas H.; Leiserson, Charles E., Rivest, Ronald L. (1990). Introduction to Algorithms (1st ed.). MIT Press and McGraw-Hill. ISBN 0-262-03151-8.

[15] The Node Distribution of the Random Waypoint Mobility Model for Wireless Ad Hoc Networks, Christian Bettstetter, Student Member, IEEE, Giovanni Resta, and Paolo Santi, IEEE TRANSACTIONS ON MOBILE COMPUTING, VOL. 2, NO. 3, Page 257- 269, JULYSEPTEMBER 2003

[16] Harding, Stephen. U.S. Army Aircraft Since 1947. Schiffer Publishing Ltd., 1997. 
[17] Richard L. Knoblauch, Martin T. Pietrucha, and Marsha Nitzburg, "Field Studies of Pedestrian Walking Speed and Start-Up Time." The Transportation Research Board's Transportation Research Record No. 1638, Pedestrian and Bicycle Research, 1996.

[18] Theodoere S. Rappaport, Wireless Communications: Principles and Practice, Second Edition, Prentice Hall: Upper Saddle River, NJ, 2002, ISBN 0-14-042232-0

[19] The Joint Tactical Radio System (JTRS) and the Army's Future Combat System (FCS): Issues for Congress, Nov. 17, 2005

\section{Authors}

Mr. Thabet Mismar is a Ph.D. student at the University of Toledo. Thabet Mismar received his M.Sc. degree in Electrical Engineering from the University of Bridgeport. He received his B.Sc. degree of Electrical Engineering from the University of Jordan. He has research interests in wireless and mobile communications.

Dr. Khaled Elleithy is the Associate Dean for Graduate Studies in the School of Engineering at the University of Bridgeport. He has research interests are in the areas of network security, mobile communications, and formal approaches for design and verification. He has published more than one hundred twenty research papers in international journals and conferences in his areas of expertise.

Dr. Elleithy is the co-chair of the International Joint Conferences on Computer, Information, and Systems Sciences, and Engineering (CISSE).
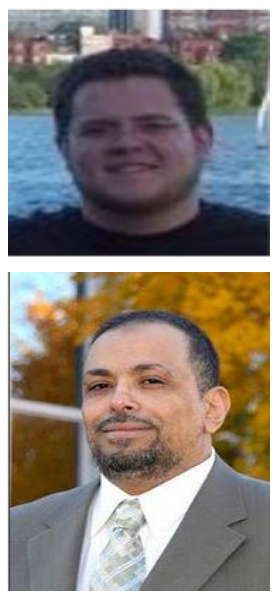

CISSE is the first Engineering/Computing and Systems Research E-Conference in the world to be completely conducted online in real-time via the internet and was successfully running for four years.

Dr. Elleithy is the editor or co-editor of 10 books published by Springer for advances on Innovations and Advanced Techniques in Systems, Computing Sciences and Software.

Dr. Elleithy received the B.Sc. degree in computer science and automatic control from Alexandria University in 1983, the MS Degree in computer networks from the same university in 1986, and the MS and Ph.D. degrees in computer science from The Center for Advanced Computer Studies in the University of Louisiana at Lafayette in 1988 and 1990, respectively.

Dr. Saikat Ray received his B.Tech. degree from Indian Institute of Technology, Guwahati, India in Electronics and Communications Engineering in 2000, and M.S. and Ph.D. degrees from Boston University in Electrical Engineering in 2002 and 2005, respectively. He spent the summers of 2001 and 2003 as interns in Fujitsu Network Communications (Acton, MA, USA) and Microsoft Research (Cambridge, UK),

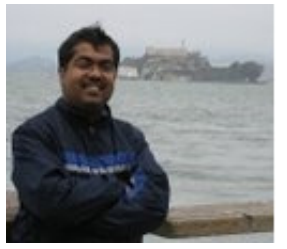
respectively.

Dr. Ray was a post-doctoral researcher in the Department of Electrical and Systems Engineering, University of Pennsylvania from September 2005 to December 2006. From January 2007 to December 2008 he worked as an Assistant Professor at University of Bridgeport. Currently he is a software engineer in the beautiful San Francisco Bay area working for Redback Networks (Ericsson). 